\title{
Screening lager yeast with higher ethyl-acetate production by adaptive laboratory evolution in high concentration of acetic acid
}

\section{$\mathrm{Xin} \mathrm{Xu}$}

Jiangnan University

Chengtuo Niu

Jiangnan University

Chunfeng Liu

Jiangnan University

Jinjing Wang

Jiangnan University

Feiyun Zheng

Jiangnan University

Qi Li ( $\square$ liqi@jiangnan.edu.cn )

The Key Laboratory of Industrial Biotechnology, Jiangnan University https://orcid.org/0000-00029429-1368

\section{Research Article}

Keywords: ACS, Alcoholic fermentation, Ethyl-acetate, Lager yeast

Posted Date: April 13th, 2021

DOl: https://doi.org/10.21203/rs.3.rs-388541/v1

License: (c) (i) This work is licensed under a Creative Commons Attribution 4.0 International License.

Read Full License 


\section{Abstract}

Ethyl-acetate is important for the flavor and aroma of the alcoholic beverages, therefore, there have been extensive efforts toward increasing its production by engineering yeast strains. In this study, we reported a new approach to breed non-genetic modified producing yeast strain with higher ethyl-acetate production for beer brewing. First, we demonstrated the positive effect of higher acetic acid concentration on inducing the expression of $A C S$. Then, we applied adaptive laboratory evolution method to evolve strain with higher expression level of ACS. As a result, we obtained several strains with increased ACS expression level as well as ethyl-acetate production. In $3 \mathrm{~L}$ scale fermentation, the optimal strain EA60 synthesized more ethyl-acetate than M14 at the same time point. At the end of fermentation, the ethylacetate production in EA60 was 21.4\% higher than M14, while the other flavor components except for acetic acid were changed in a moderate degree, indicating this strain had a bright prospect in industrial application. Moreover, this study also indicated that ACS1 played a more important role in increasing the acetic acid tolerance of yeast, while ACS2 contributed to the synthesis of cytosol acetyl-CoA, thereby facilitating the production of ethyl-acetate during fermentation.

\section{Introduction}

During alcoholic fermentation, yeast cells produced a wide range of aroma-active components which greatly affected the flavor of fermented alcoholic beverages (Bloem et al. 2016; Verstrepen et al. 2003). Of all these components, esters are considered as the most important aroma elements for their contributions to the fruity and flowery notes (Pires et al. 2014). According to the compositions, these esters could be categorized as the acetate esters and the medium-chain fatty acid ethyl esters. In the beer, acetate ester, especially for ethyl-acetate, serve as the main contributor to the flavor and fragrance since the small size and lipophilic nature allow it to easily diffuse from the cytoplasm into the extracellular medium (Dzialo et al. 2017; Nykänen et al. 1977; Pires et al. 2014). It is now generally accepted that high gravity or high concentration of free amino nitrogen of wort media greatly increased the final production of ethyl-acetate in the industrial fermentations (Lei et al. 2013a; Lei et al. 2013b; Peddie 1990). Furthermore, increasing the fermentation temperatures or reducing the dissolved oxygen were also reported contributing to the production of ethyl-acetate in beer (Fujii et al. 1997; Saerens et al. 2008). Nonetheless, these effects varied given the differences in genetic backgrounds of yeast.

In the recent decades, the mechanism for ethyl-acetate biosynthesis in yeast was elucidated. Alcohol acyltransferases (AATases) played a vital role in ethyl-acetate synthesis, and higher expression level of these AATases increased the production of ethyl-acetate. On the other hand, the substrate availability, especially for acetyl-CoA, is also important for the production of ethyl-acetate. Thus simultaneously engineering the AATase activity and acetyl-CoA availability could enhance the production of ethyl-acetate (Lilly et al. 2006; Mason and Dufour 2000; Verstrepen et al. 2003; Yoshimoto et al. 1998). However, due to the safety concerns, those genetic engineered strains were rarely applied in beer industries. Therefore, it is of great necessity to trigger the expression of these genes spontaneously to increase the production of ethyl-acetate in industrial producing strains. 
The adaptive evolutionary engineering generally treats the cells under a certain pressure by repeated or prolonged cultivation until they have improved characteristics. However, the expression level of genes was tightly regulated for optimal growth in the organism's natural habitat. Therefore, it is important to design a specific pressure to trigger the expression of ACS or ATF. Since a recent study reported that overexpression of ACS2 could increase acetic acid tolerance in Saccharomyces cerevisiae (Ding et al. 2015), we assumed that higher concentrations of acetic acid can trigger the expression of ACS2 as well as ACS1 in turn. Therefore, in this study, we will first investigate whether higher concentration of acetic acid could induce the expression of ACS genes. Then adaptive laboratory evolution (ALE) would be performed to evolve the strain with upregulated expression level of ACS genes. Next, the ethyl-acetate production and other fermentation performance of the evolved strain would be compared to the parental strain in a $3 \mathrm{~L}$ scale beer fermentation to evaluate the potential of the evolved strain in industrial application. Finally, the differential roles of ACS1 and ACS2 in ethyl-acetate synthesis during fermentation were elucidated. This work will provide a new approach to breeding non-genetic modified lager yeast with higher ethyl-acetate production, thereby improving the beer quality in flavor.

\section{Materials And Methods}

\section{Strains and culture conditions}

Strains used in this study were listed in Table 1. The lager yeast (Saccharomyces pastorinous) M14 (Liu et al. 2017) was used as the background strain, while M-ACS1 and M-ACS2 were constructed by overexpressing corresponding gene using an expression plasmid YEP352 under the control of PGK1 promotor (Xu et al. 2018a). Primers used for amplification of ACS1 and ACS2 were listed in Table 2. EA60 was the evolved strain of M14 using the ALE method. The yeast strains were cultured in YPD medium or wort medium, supplemented with $\mathrm{G} 418$ when necessary, at $28^{\circ} \mathrm{C}$.

\section{Quantitative Real-Time-PCR analysis}

Yeasts cells were collected at the mid-log phase and centrifuged at $3,000 \times \mathrm{g}$ for 5 minutes at $4{ }^{\circ} \mathrm{C}$. Then the total RNA was extracted from cells using UNIQ-10 column Trizol total RNA isolation kit (Sangon Biotech), following the manufacturer's instruction. The three RNAs from 3 parallel samples were mixed and a total of $1 \mu \mathrm{g}$ of RNA was used to synthesize the first-strand cDNA using PrimeScript RT reagent Kit with gDNA Eraser (Perfect Real Time, Takara, Dalian, China). Then cDNA samples were diluted in water at a ratio of 1:5, and qRT-PCR was performed with the SYBR Premix Ex Taq ${ }^{\mathrm{TM}} \|$ kit (Takara, Dalian, China) in a LightCycler 480 PCR system (Roche Diagnostics, Shanghai, China) in triplicate (Xu et al. 2018b). ACT1 was used as a standard control to normalize the gene expression. Primers used for RT-qPCR were listed in Table 2

\section{Cellular acetyl-CoA level measurement}

To measure the cellular acetyl-CoA level, $2 \mathrm{~mL}$ collected yeasts at the mid-log phase were quenched and then mixed with $2 \mathrm{~mL}$ boiling ethanol for $15 \mathrm{~min}$ to release the metabolite. Next, the mixture was 
centrifuged at $12,000 \times g$ for 10 minutes at $4{ }^{\circ} \mathrm{C}$ and the supernatant was dried by the vacuum freeze dryer. Finally, $200 \mu \mathrm{L} \mathrm{ddH_{2 } O}$ was used to re-suspend the metabolite and the cetyl-CoA was detected by LCMS/MS (Armando et al. 2012).

\section{Beer fermentation}

To prepare wort, pure Canadian malt (Supertime, China) was crashed and mixed with water in a ratio of $4.5: 1(\mathrm{~kg} / \mathrm{kg})$. The mash profile was processed as follows: $48^{\circ} \mathrm{C}, 30 \mathrm{~min} ; 63^{\circ} \mathrm{C}, 60 \mathrm{~min} ; 72^{\circ} \mathrm{C}, 30$ $\min$ and $78^{\circ} \mathrm{C}, 10 \mathrm{~min}$. Then the wort was filtered and boiled for $60 \mathrm{~min}$ with hops $(0.3 \%$ ). Finally, the boiled mixture was filtered again, adjusted to $11^{\circ} \mathrm{P}$ and sterilized at $105^{\circ} \mathrm{C}$ for $10 \mathrm{~min}$.

The main fermentation process was detailed described in our previous works (Xu et al. 2018b). In brief, the yeast strains were initially pre-cultured in $100 \mathrm{~mL}$ wort at $28^{\circ} \mathrm{C}$ for 2 days and then collected after $12-\mathrm{h}$ natural sedimentation at $4^{\circ} \mathrm{C}$. After this, yeast was inoculated to $300 \mathrm{~mL}$ wort media with a cell density around $10^{7}$ cells $/ \mathrm{mL}$ for primary fermentation. Air locks were used to close the flasks, which ensured the exclusion of oxygen but allowed for the release of $\mathrm{CO}_{2}$. The main fermentation process proceeded at $11^{\circ} \mathrm{C}$ for 7 days. The weight of the total fermentation equipment was recoded every day to calculate the weight loss. Three biological repeats of each experiment were performed. Strain M14 and EA60 were also used to perform a $3 \mathrm{~L}$ scale fermentation with $2 \mathrm{~L}$ wort media at $11^{\circ} \mathrm{C}$ for 10 days and two parallel experiments of each strain were carried out.

\section{Adaptive laboratory evolution}

The wort media supplied with $0.6 \%$ acetic acid was used throughout this section. Strain M14 was pre-cultured in the wort media at $28^{\circ} \mathrm{C}$ for 2 days, then cells were transferred into fresh media with $0.6 \%$ acetic acid every $24 \mathrm{~h}$ with an initial $\mathrm{OD}_{600}$ value at 0.1 . During adaptive evolution, cells were collected at day 10 , day 20 , day 30 , day 40 , day 50 and day 60 , and a detailed cell growth curve was monitored.

\section{By-products analysis}

Organic acids were analyzed using HPLC (Chromaster, Hitachi Ltd., Shanghai, China) with an Atlantis 100-C18 column ( $5 \mathrm{~mm}, 4.6^{\prime} 250 \mathrm{~mm}$ ) (Waters, Shanghai, China) and an ultraviolet-visible detector. The mobile phase consisted of $20 \mathrm{mmol} / \mathrm{L} \mathrm{KH}_{2} \mathrm{PO}_{4}$, with the flow rate of $0.5 \mathrm{~mL} / \mathrm{min}$, and the column temperature was maintained at $25^{\circ} \mathrm{C}$. Alcohols and esters were measured using headspace gas chromatography (GC-2010 PerkinElmer TurboMatrix 16, Shimadzu, China) with a Rtx-Wax column (30 m, $0.32 \mathrm{~mm}, 0.25 \mathrm{~mm}$ ), using 3-heptanone as an internal standard. Diacetyl content was measured using a diacetyl distiller based on the Chinese National standard for beer analysis, which had been described clearly (Shi et al. 2017). The ethanol content, apparent extract and attenuation were determined by Anton Paar Alcohol meter equipped with Alcolyzer Beer ME and DMA 4500M.

\section{Results}




\section{Acetic acid triggers the expression of ACS}

To evaluate the impact of acetic acid on the expression of ACS gene, we treated the yeast in wort media with different concentration of acetic acid. The cell growth of M14 was slightly affected when the acetic acid concentration was lower than $0.5 \%$, while a dramatic growth defect was observed when the acetic acid concentration reached to $0.5 \%$ (Fig. $1 \mathrm{a}$ ). When the acetic acid concentration reached $0.8 \%$, the cell growth of M14 was completely inhibited (Fig. 1a). Meanwhile, we collected the cells and measured the mRNA level of $A C S 1$ and $A C S 2$ in M14 under these conditions by RT-qPCR. The results indicated that the expression levels of $A C S 1$ and $A C S 2$ in M14 were upregulated significantly when the acetic acid concentration was higher than $0.3 \%$ (Fig. 1b). Moreover, the relative expression level of ACS1 was higher than that of ACS2. To explore whether ACS1 played a more important role in acetic acid tolerance, we overexpressed ACS1 and ACS2 in M14 respectively (Fig. 1c) and evaluated the growth capacity of the generated strains (M-ACS1 and M-ACS2) under $0.6 \%$ acetic acid conditions. As a result, the cell growth of M-ACS1 was obviously better than that of M-ACS2 (Fig. 1d), indicating ACS1 was more important than ACS2 in relieving the pressure of acetic acid on cell growth. Nonetheless, the cell growth of M-ACS2 was still better than that of M14, which was in line with the previous work (Ding et al. 2015).

\section{Breeding lager yeast with higher acetyl-acetate production using ALE}

Then we used the ALE method to evolve the strain M14 under the pressure of $0.6 \%$ acetic acid. Within this procedure, the cell growth was gradually improved during evolution (Fig. 2a). After 60 runs transfer, we ended it and separated several single colonies on the plate. We randomly picked 10 colonies and performed beer fermentation experiments with these strains. The total weight loss of these strains at the end of main fermentation was similar to that of M14 (Fig. 2b), indicating the fermentation performance was not much affected. At the end of main fermentation, the ethyl-acetate productions of these strains were obvious increased (Fig. 2c). The optimal strain No.5 (called EA60 in the later text) produced 18.53 $\mathrm{mg} / \mathrm{L}$ ethyl-acetate, which was improved by $40.3 \%$ when compared with parental strain M14. RT-qPCR results showed that the expression level of ACS1 and ACS2 in EA60 were upregulated by 3.7 and 2.1-fold respectively (Fig. 2d), which was in line with our expectation.

Next, we performed a $3 \mathrm{~L}$ scale fermentation with EA60 to evaluate its potential in industrial application. After inoculation into wort media, the cell growth of EA60 was superior to M14, resulting the cell amount of EA60 was higher than that of M14 (Fig. 3a). During the fermentation, EA60 consumed the fermented sugars quicker than M14, which reflecting on the apparent extract (Fig. 3b). Meanwhile, EA60 synthesized more ethyl-acetate than M14 at the same time course. At the end of fermentation, the ethyl-acetate production of EA60 was approximate at $18.60 \mathrm{mg} / \mathrm{L}$, which was $21.4 \%$ higher than M14 (Table 3). The ethanol content of EA60 was decreased from $4.68 \%$ to $4.47 \%$ while the real attenuation was increased from $65.21 \%$ to $66.04 \%$. In terms of the flavor components, we found the acetate was increased by $57.6 \%$ in EA60 while the other components such as higher alcohols and organic acid were changed in a moderate degree. Overall, these results suggested that the fermentation performance and the aroma profile of EA60 could meet the requirement for industrial production. 


\section{The role of $A C S 1$ and $A C S 2$ on ethyl-acetate production during fermentation}

In EA60, the expression of ACS1 and ACS2 were both upregulated. Therefore, we would like to investigate which one contribute more to ethyl-acetate synthesis under beer fermentation conditions. We again used the engineered strains M-ACS1 and M-ACS2 to explore this question. We measured the cellular acetyl-CoA contents in the engineered strains at the mid-log phase and found that the cellular acetyl-CoA contents in these two strains were increased by $10.4 \%$ and $16.7 \%$, respectively (Fig. 4a). However, during fermentation, we found that the production of ethyl-acetate of M-ACS1 was similar to that of M14 at the early fermentation but slightly higher than M14 at the later phase. By contrast, the ethyl-acetate synthesis of M-ACS2 was always higher than that of M14 at the same time course (Fig. 4b). At the end of fermentation, the ethyl-acetate production for M14, M-ACS1 and M-ACS2 was 13.27, 14.10 and 16.84 $\mathrm{mg} / \mathrm{L}$ respectively $(p<0.05)$. These results suggested that ACS2 was the main contributor to the synthesis of ethyl-acetate during fermentation, while ACS1 contributed less despite the increased cellular acetyl-CoA level.

\section{Discussions}

Esters synthesized by lager yeast are important to the beer flavor, therefore breeding lager yeast with higher levels of these components is always a hot point in beer industry. Owing to the development of metabolic approaches and technologies, great efforts had been made to engineer the Saccharomyces cerevisiae or lager yeast with higher levels of ethyl-acetate production. However, in consideration of the consumers' acceptance and the policies regarding food safety, those genetic modified strains could not be applied in beer industry in China. Therefore building a bridge between biological knowledge and practical application is of great importance.

The availability of enzyme and substrate was the limiting factor for the synthesis of ethyl-acetate, thus increasing their abundances would be a promising strategy. In this study, we assumed that higher concentration of acetic acid could trigger the expression of ACS. Then through ALE, the expression of those genes would be upregulated spontaneously, resulting the evolved strain possessed higher acetylCoA level as well as higher ethyl-acetate production. As a result, we indeed found the expression of ACS1 and ACS2 in M14 were both increasingly upregulated along with the increased concentration of acetic acid. Meanwhile we found the upregulated level of ACS1 was higher than that of ACS2. As Acs1p has a 30-fold higher affinity to acetate than Acs2p (van den Berg et al. 1996), higher expression level of ACS1 may contribute more to relieve the pressure of acetic acid. This reason could be supported by the result that the cell growth of M-ACS1 in $0.6 \%$ acetic acid was better than M-ASC2 and M14. The previous work only reported that overexpression of ACS2 could increase the yeast tolerance for exogenous acetic acid. Here, our results suggested that both ACS1 and ACS2 could confer yeast with such capacity and ACS1 had a better effect.

Through ALE, we obtained several strains with higher production ethyl-acetate. Higher expression level of ACS in EA60 could explain the higher production of ethyl-acetate to a certain degree, but a further 
comparative genomic analysis between strain M14 and EA60 was still needed to dissect the underlying regulation mechanism. As ACS enhanced the carbon flux toward acetyl-CoA (Lian et al. 2018; Lian et al. 2014), the intermediate product (acetaldehyde and acetate) was thereupon simultaneously increased in the final fermentation liquid. Whereas, the production of another acetate ester, isoamyl acetate, was not increased in EA60. This may due to the production of isoamyl acetate was subject to isoamyl alcohol content (Yoshioka and Hashimoto 1984).

Of note, the impact of $A C S 1$ and $A C S 2$ on ethyl-acetate synthesis was quite different under fermentation conditions. The cellular acetyl-CoA levels in M-ACS1 and M-ACS2 were both increased while the increased ethyl-acetate production of M-ACS1 was lower than expected. Such a phenomenon could be explained by the different distribution of Acs1p and Acs2p in yeast. A systematic study suggested Acs $2 p$ was exclusively located in the cytosol while Acs1p was subjected to dual distribution, e.g. to the peroxisomes under glucose repressing conditions but to the cytoplasm under de-repressing conditions (Fig. 4c) (Chen et al. 2012). Under the wort fermentation conditions where a plenty of fermented sugars exist, the Acs $1 p$ is of high likely to distributed to peroxisomes but not to cytosol. The lipid synthesis for cell growth preferred cytosolic acetyl-CoA, as a result, we found that the cell growth of M-ACS1 was similar to that of M14, while M-ACS2 showed a remarkable increase in cell growth (Fig. 4d). This result suggested that ACS1 contribute to acetyl-CoA synthesis in the peroxisomes under fermentation conditions. Further, as AATase is located in the endoplasmic reticulum or lipid droplets (Fig. 4c) (Lin and Wheeldon 2014), those increased acetyl-CoA could not serve as effective substrate for the synthesis of ethyl-acetate.

Taken together, this study demonstrated that higher concentration of acetic acid could trigger the expression of $A C S$, and using ALE method, a non-genetic modified strain EA60 was obtained with higher level of ethyl-acetate for industrial application.

\section{Declarations}

\section{Acknowledgments}

This work was financially supported by the Priority Academic Program Development of Jiangsu Higher Education Institutions (PAPD), Program of Introducing Talents of Discipline to Universities (no. 111-2-06), the National High Technology Research and Development program 863 (no. 2018YFD0400403) and the National Science Foundation (no. 31771963 and no. 31901626).

\section{Conflict of interest}

The authors declare that they have no conflict of interest.

\section{Ethical approval}

This article does not contain any studies with human participants or animals performed by any of the authors. 


\section{References}

Armando JW, Boghigian BA, Pfeifer BA (2012) LC-MS/MS quantification of short-chain acyl-CoA's in Escherichia coli demonstrates versatile propionyl-CoA synthetase substrate specificity. Lett Appl Microbiol 54(2):140-8 doi:10.1111/j.1472-765X.2011.03184.x

Bloem A, Sanchez I, Dequin S, Camarasa C (2016) Metabolic impact of redox cofactor perturbations on the formation of aroma compounds in Saccharomyces cerevisiae. Appl Environ Microbiol 82(1):174-83 doi:10.1128/aem.02429-15

Chen Y, Siewers V, Nielsen J (2012) Profiling of cytosolic and peroxisomal acetyl-coa metabolism in Saccharomyces cerevisiae. PLoS One 7(8):e42475 doi:10.1371/journal.pone.0042475

Ding J, Holzwarth G, Penner MH, Patton-Vogt J, Bakalinsky AT (2015) Overexpression of acetyl-CoA synthetase in Saccharomyces cerevisiae increases acetic acid tolerance. FEMS Microbiol Lett 362(3):1-7 doi:10.1093/femsle/fnu042

Dzialo MC, Park R, Steensels J, Lievens B, Verstrepen KJ (2017) Physiology, ecology and industrial applications of aroma formation in yeast. FEMS Microbiol Rev 41(Supp_1):S95-s128 doi:10.1093/femsre/fux031

Fujii T, Kobayashi O, Yoshimoto H, Furukawa S, Tamai Y (1997) Effect of aeration and unsaturated fatty acids on expression of the Saccharomyces cerevisiae alcohol acetyltransferase gene. Appl Environ Microbiol 63(3):910-915

Lei H, Li H, Mo F, Zheng L, Zhao H, Zhao M (2013a) Effects of Lys and His supplementations on the regulation of nitrogen metabolism in lager yeast. Appl Microbiol Biotechnol 97(20):8913-8921

Lei H, Zheng L, Wang C, Zhao H, Zhao M (2013b) Effects of worts treated with proteases on the assimilation of free amino acids and fermentation performance of lager yeast. Int $\mathrm{J}$ Food Microbiol 161(2):76-83

Lian J, Mishra S, Zhao H (2018) Recent advances in metabolic engineering of Saccharomyces cerevisiae: New tools and their applications. Metab Eng 50:85-108 doi:10.1016/j.ymben.2018.04.011

Lian J, Si T, Nair NU, Zhao H (2014) Design and construction of acetyl-CoA overproducing Saccharomyces cerevisiae strains. Metab Eng 24:139-49 doi:10.1016/j.ymben.2014.05.010

Lilly M, Bauer FF, Lambrechts MG, Swiegers JH, Cozzolino D, Pretorius IS (2006) The effect of increased yeast alcohol acetyltransferase and esterase activity on the flavour profiles of wine and distillates. Yeast 23(9):641-59 doi:10.1002/yea.1382

Lin JL, Wheeldon I (2014) Dual N- and C-terminal helices are required for endoplasmic reticulum and lipid droplet association of alcohol acetyltransferases in Saccharomyces cerevisiae. PLoS One 9(8):e104141 
Liu C, Li Q, Niu C, Zheng F, Li Y, Zhao Y, Yin X (2017) Genome sequence of the lager-brewing yeast Saccharomyces sp. strain m14, used in the high-gravity brewing industry in China. Genome Announc 5(43) doi:10.1128/genomeA.01194-17

Mason AB, Dufour JP (2000) Alcohol acetyltransferases and the significance of ester synthesis in yeast. Yeast 16(14):1287-98 doi:10.1002/1097-0061(200010)16:14<1287::aid-yea613>3.0.co;2-i

Nykänen L, Nykänen I, Suomalainen H (1977) Distribution of esters produced during sugar fermentation between the yeast cell and the medium. Journal of the Institute of Brewing 83(1):32-34

Peddie HA (1990) Ester formation in brewery fermentations. Journal of the Institute of Brewing 96(5):327331

Pires EJ, Teixeira JA, Branyik T, Vicente AA (2014) Yeast: the soul of beer's aroma--a review of flavouractive esters and higher alcohols produced by the brewing yeast. Appl Microbiol Biotechnol 98(5):193749 doi:10.1007/s00253-013-5470-0

Saerens S, Verbelen P, Vanbeneden N, Thevelein J, Delvaux F (2008) Monitoring the influence of highgravity brewing and fermentation temperature on flavour formation by analysis of gene expression levels in brewing yeast. Appl Microbiol Biotechnol 80(6):1039-1051

Shi TT, Li P, Chen SJ, Chen YF, Guo XW, Xiao DG (2017) Reduced production of diacetyl by overexpressing $B D H 2$ gene and $I L V 5$ gene in yeast of the lager brewers with one $I L V 2$ allelic gene deleted. $J$ Ind Microbiol Biotechnol 44(3):397-405 doi:10.1007/s10295-017-1903-6

van den Berg MA, de Jong-Gubbels P, Kortland CJ, van Dijken JP, Pronk JT, Steensma HY (1996) The two acetyl-coenzyme A synthetases of Saccharomyces cerevisiae differ with respect to kinetic properties and transcriptional regulation. J Biol Chem 271(46):28953-9

Verstrepen KJ, Van Laere SD, Vanderhaegen BM, Derdelinckx G, Dufour JP, Pretorius IS, Winderickx J, Thevelein JM, Delvaux FR (2003) Expression levels of the yeast alcohol acetyltransferase genes ATF1, $L g-A T F 1$, and ATF2 control the formation of a broad range of volatile esters. Appl Environ Microbiol 69(9):5228-37

Xu X, Liu C, Niu C, Wang J, Zheng F, Li Y, Li Q (2018a) Rationally designed perturbation factor drives evolution in Saccharomyces cerevisiae for industrial application. J Ind Microbiol Biotechnol 45(10):869880 doi:10.1007/s10295-018-2057-x

Xu X, Wang J, Bao M, Niu C, Liu C, Zheng F, Li Y, Li Q (2018b) Reverse metabolic engineering in lager yeast: impact of the $\mathrm{NADH} / \mathrm{NAD}^{+}$ratio on acetaldehyde production during the brewing process. Appl Microbiol Biotechnol doi:10.1007/s00253-018-9517-0 
Yoshimoto H, Fujiwara D, Momma T, Ito C, Sone H, Kaneko Y, Tamai Y (1998) Characterization of the ATF1 and Lg-ATF1 genes encoding alcohol acetyltransferases in the bottom fermenting yeast Saccharomyces pastorianus. J Ferment Bioeng 86(1):15-20

Yoshioka K, Hashimoto N (1984) Acetyl-CoA of brewers' yeast and formation of acetate esters. Agricultural and Biological Chemistry 48(1):207-209 doi:10.1080/00021369.1984.10866117

\section{Tables}

Table 1. Strains used in this study

\begin{tabular}{lll}
\hline Strains & Description & Resource \\
\hline M14 & Lager brewers' yeast & Our lab \\
M-ACS1 & M14 derivate, overexpressing ACS1 under the PGK1 promotor & This study \\
M-ACS2 & M14 derivate, overexpressing ACS2 under the PGK1 promotor & This study \\
\hline
\end{tabular}

Table 2. Primers used in this study

\begin{tabular}{ll}
\hline Primer & Sequence \\
\hline Plasmid construction & \\
ACS1F & ctagctagcATGTCGCCCTCTGC \\
ACS1R & ccgctcgagTTACAACTTGACCG \\
ACS2F & ctagctagc ATGACAATCA AGG \\
ACS2R & ccgctcgag TTATTTCTTTTTTGAGAG \\
RT-qPCR & \\
ACS1(qPCR) & GTGCTGACTTACTCTATG/ AGGTTATGATTGCTTCTG \\
ACS2(qPCR) & TACTGAAGGTATTCCAATG/ AGGTAGGTAAGTTCTCTG \\
\hline
\end{tabular}

Table 3. Parameters measured in the pilot scale fermentation. The pilot scale fermentation was performed in a 3 L Erlenmeyer flask with $2 \mathrm{~L} 11^{\circ} \mathrm{P}$ wort at $11^{\circ} \mathrm{C}$ for 10 days with two repeats and the values were means \pm SD of two independent experiments 


\begin{tabular}{lcc}
\hline & M14 & EA60 \\
\hline Real attenuation (\%) & $65.21 \pm 0.42$ & $66.04 \pm 0.51$ \\
Diacetyl (mg/L) & $0.04 \pm 0.005$ & $0.04 \pm 0.007$ \\
Ethanol (\%) & $4.68 \pm 0.1$ & $4.47 \pm 0.1$ \\
Ethyl-acetate (mg/L) & $15.32 \pm 0.11$ & $18.60 \pm 0.17$ \\
Isoamyl acetate (mg/L) & $0.53 \pm 0.03$ & $0.50 \pm 0.05$ \\
$n$-Propanol (mg/L) & $8.23 \pm 0.12$ & $8.57 \pm 0.27$ \\
Isoamyl alcohol (mg/L) & $40.62 \pm 1.12$ & $38.91 \pm 0.98$ \\
Isobutanol (mg/L) & $6.43 \pm 0.04$ & $6.71 \pm 0.07$ \\
Acetate (mg/L) & $99.01 \pm 2.13$ & $156.0 \pm 5.11$ \\
Acetaldehyde (mg/L) & $8.65 \pm 0.12$ & $9.21 \pm 0.05$ \\
Malate (mg/L) & $128.11 \pm 5.27$ & $119.43 \pm 6.41$ \\
Citrate (mg/L) & $97.26 \pm 1.28$ & $100.33 \pm 0.85$ \\
Succinate (mg/L) & $162.17 \pm 3.91$ & $157.26 \pm 5.37$ \\
\hline
\end{tabular}

Figures 
a

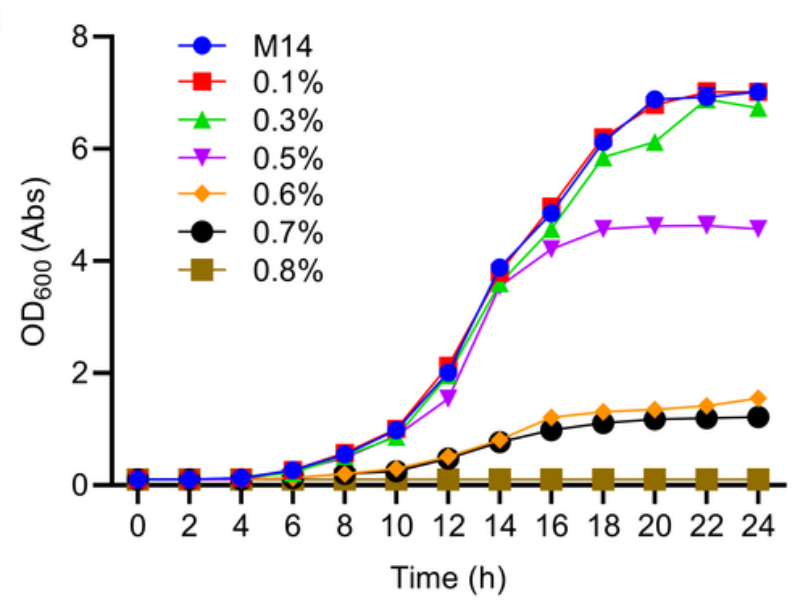

C

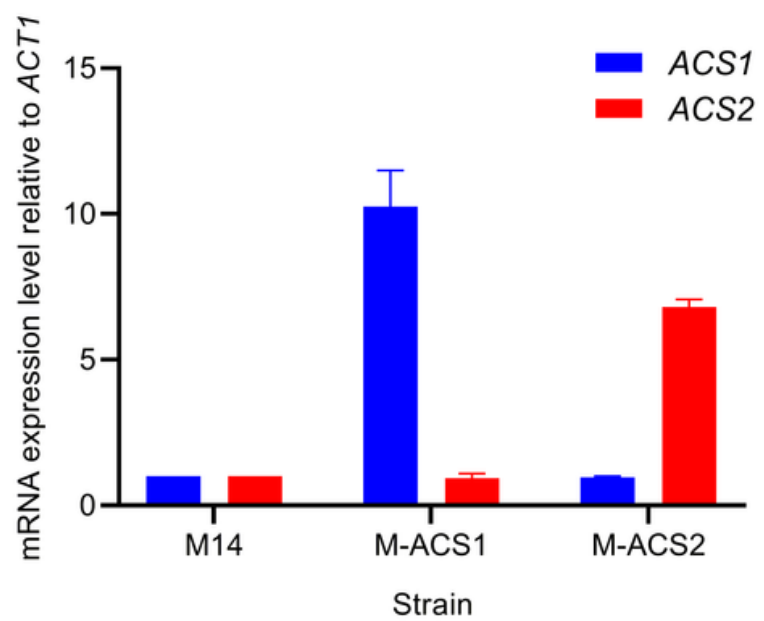

b

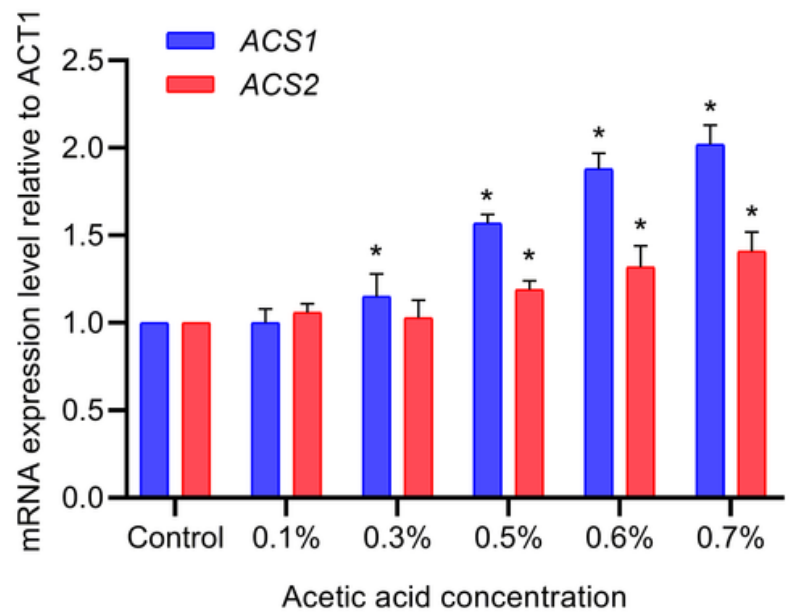

d

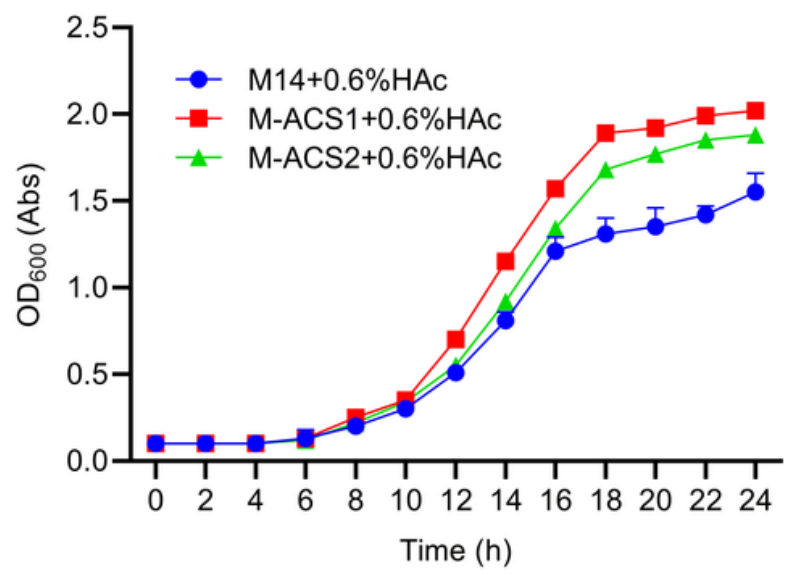

Figure 1

Acetic acid triggered the expression of ACS and higher expression levels of ACS confer the yeast with acetic acid tolerance. (a) The cell growth of parental strain M14 in the wort media with different concentration of acetic acid; (b) The relative expression level of ACS1 and ACS2 in M14 under different concentration of acetic acid; (c) The relative expression level of ACS1 and ACS2 in the engineered strains M-ACS1 and M-ACS2 which overexpression ACS1 and ACS2 respectively; (d) The cell growth of M-ACS1 and M-ACS2 in wort media supplied with $0.6 \%$ acetic acid. 

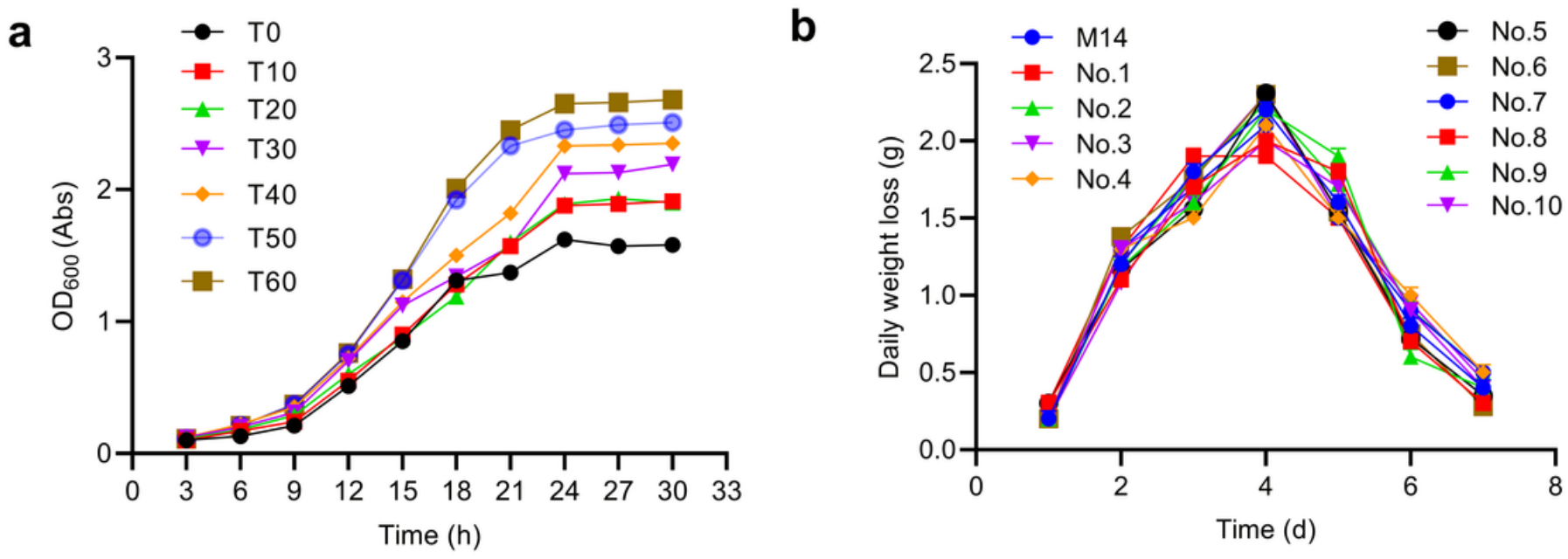

C

d
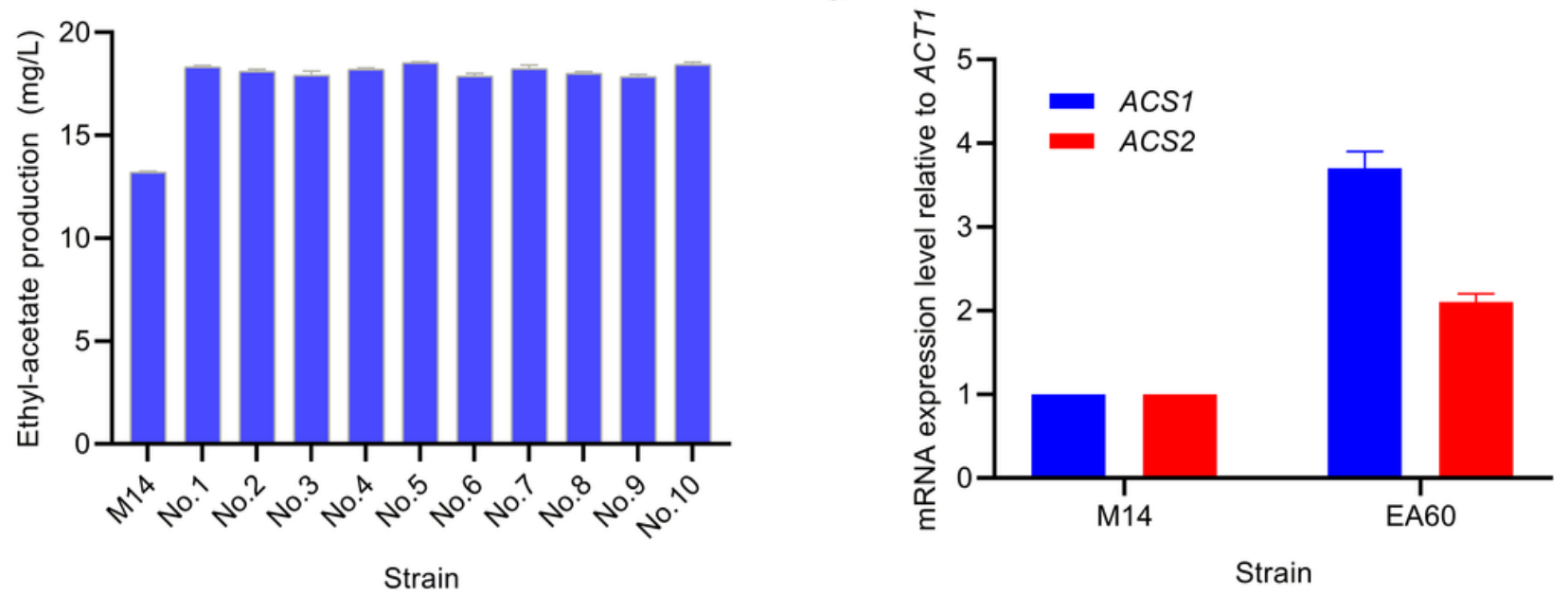

Figure 2

Breeding lager yeast with higher ethyl-acetate production. (a) The cell growth of the strains under $0.6 \%$ acetic acid during ALE process; (b) The daily weight loss (fermentation rate) of randomly picked strains seperated from the evolved strains which were transferred by 60 runs; (c) The ethyl-acetate production of the randomly picked strains; (d) The relatively expression level of ACS1 and ACS2 in the optimal strain EA60. 
a

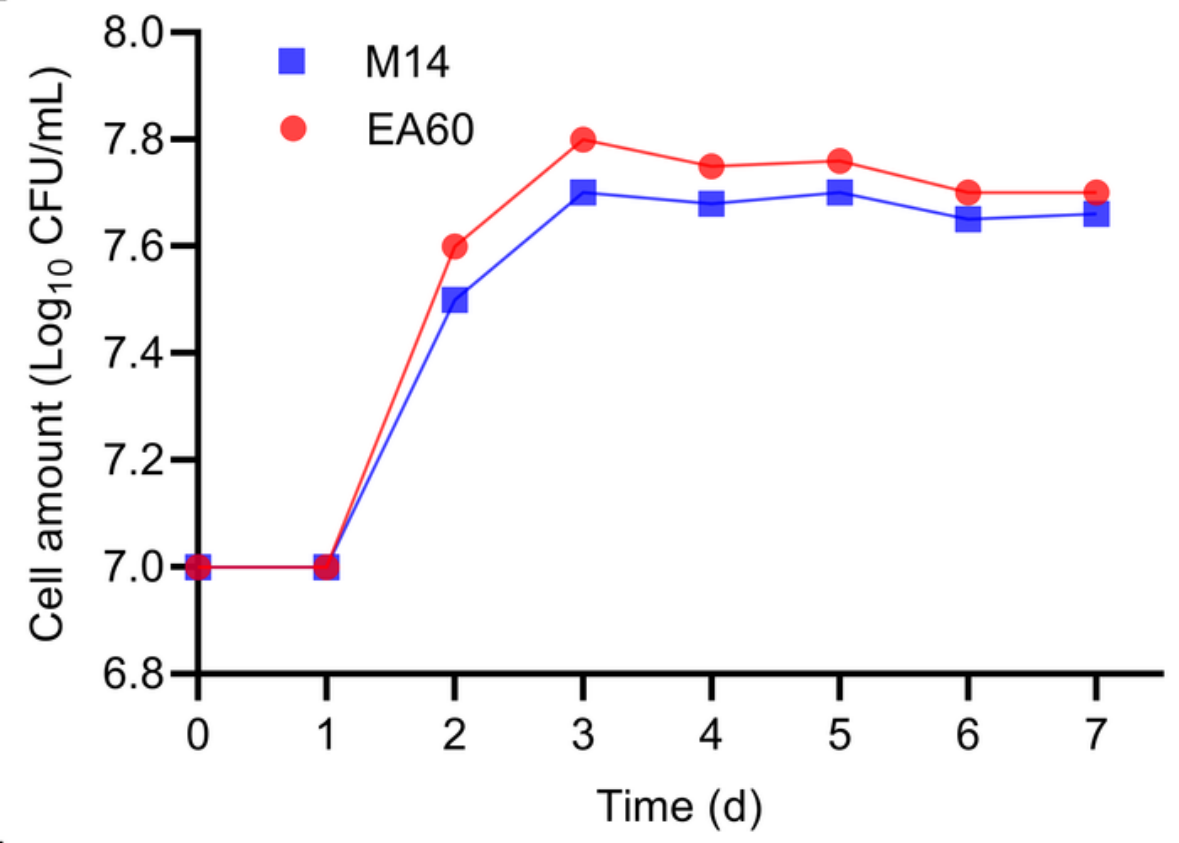

b

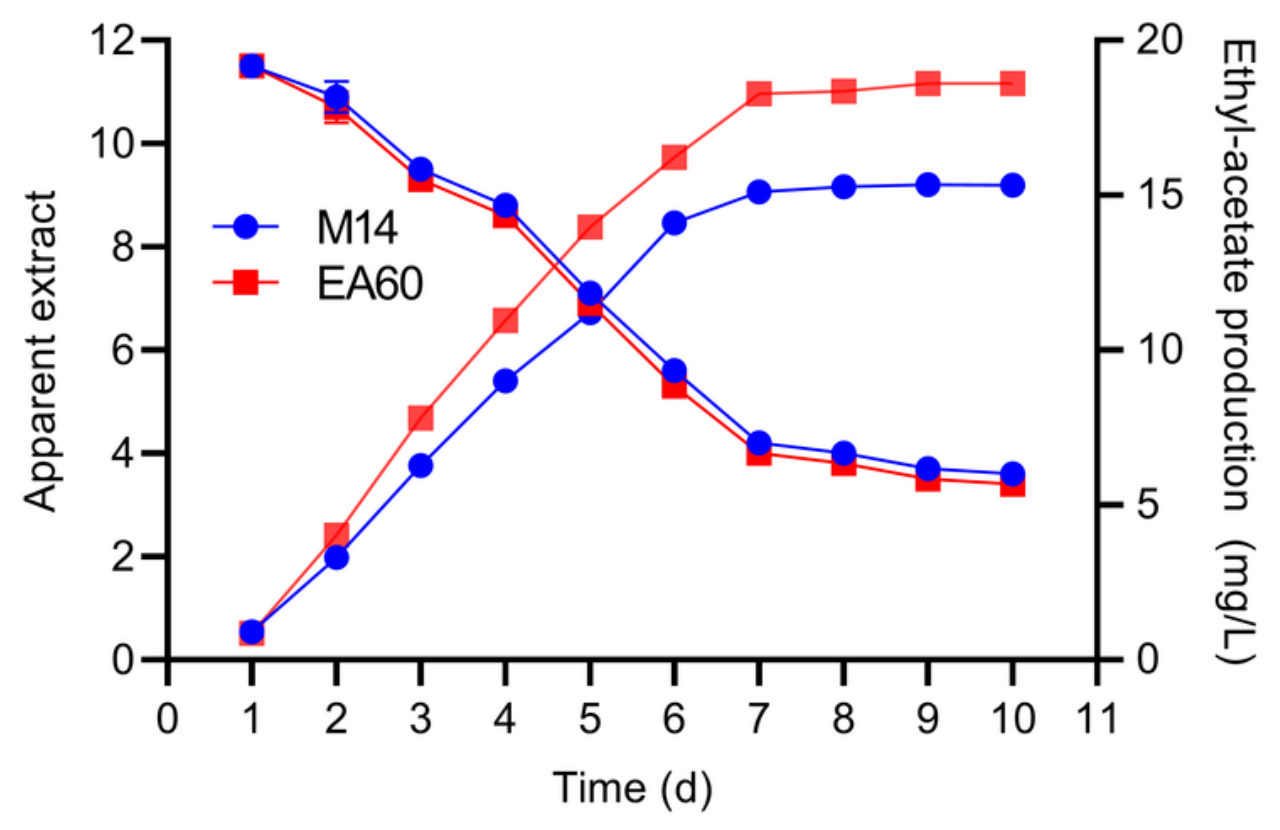

Figure 3

Time course of M14 and EA60 during alcoholic fermentation. (a) The cell amount during fermentation; (b) The dynamic changes of apparent extract and ethyl-acetate contents. The brewing experiment was carried in a $3 \mathrm{~L}$ Erlenmeyer flask with $2 \mathrm{~L} 11^{\circ} \mathrm{P}$ wort at $11^{\circ} \mathrm{C}$ for 10 days with two repeats and the results were shown as means \pm SD. 
$\mathbf{a}$

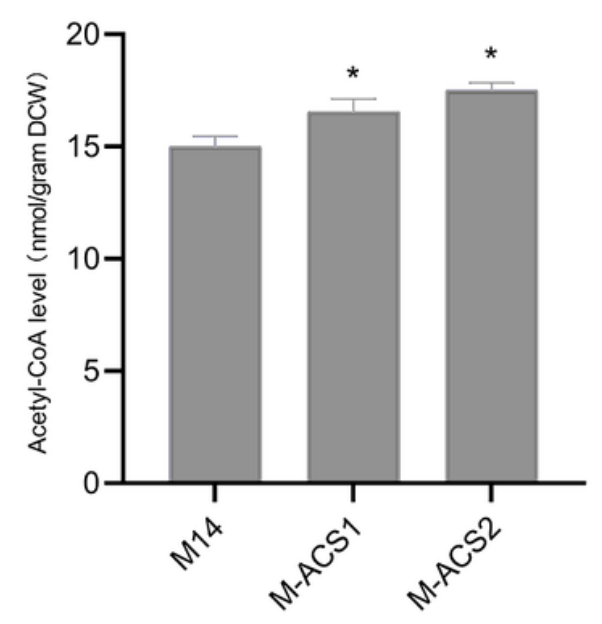

Strain

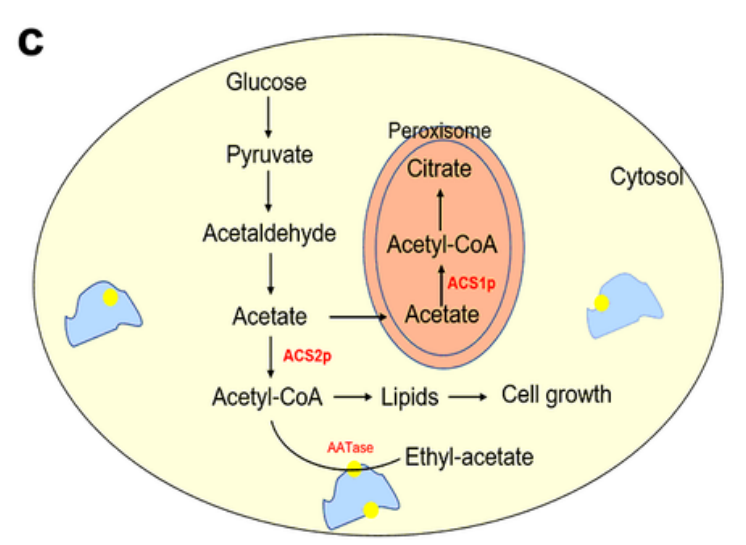

b

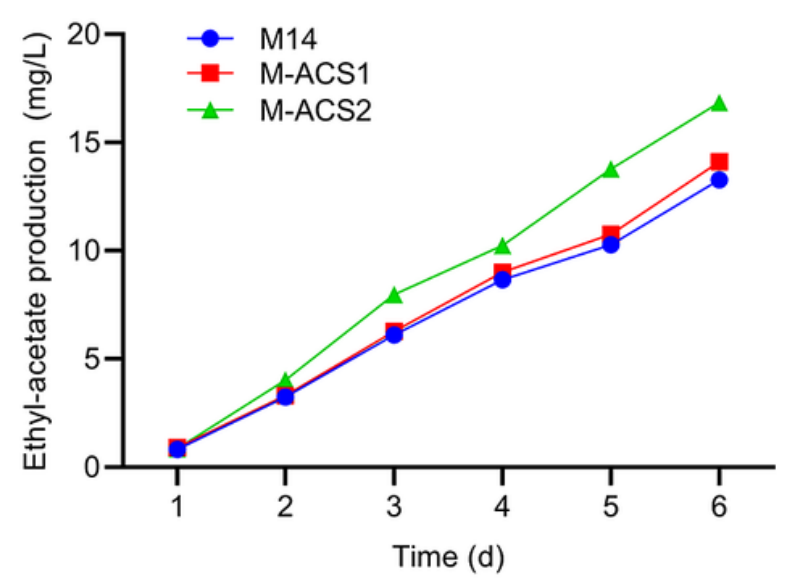

d

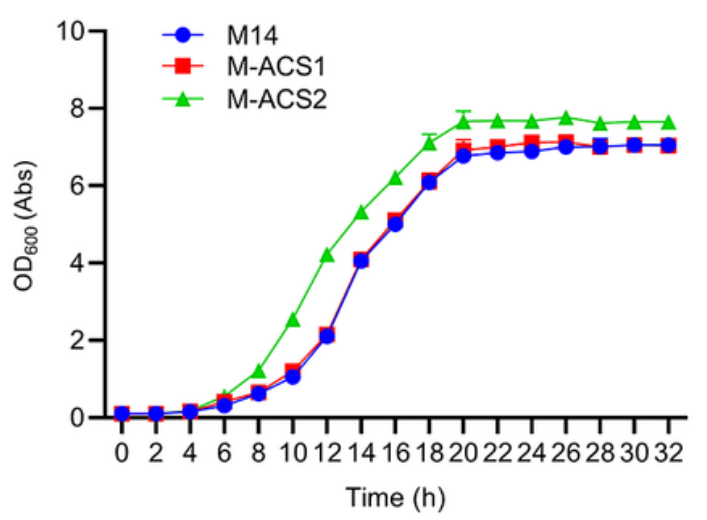

\section{Figure 4}

The different contribution of ACS1 and ACS2 to ethyl-acetate synthesis during alcoholic fermentation. (a) The cellular acetyl-CoA level of strain M14, M-ACS1 and M-ACS2; (b) The ethyl-acetate production; (c) The putative distribution of Acs1p and Acs2p in yeast cells; (d) The growth curve of strain M14, M-ACS1 and M-ACS2. 